\section{Ergebnisse des Expertengesprächs «Enterale Ernährungstherapie» der Deutschen Gesellschaft für Ernährungsmedizin (DGEM), Bonn, 14. Juni $1999^{1}$}

\section{Abschließendes Eckpunktepapier}

Der Bedarf an enteraler Ernährung ist groß. Die Sozialgesetzgebung, die Ausbildung der Ärzte sowie die Vergütung der Leistungen werden jedoch dem Anspruch einer adäquaten, bedarfsgerechten enteralen Ernährungstherapie nicht gerecht. Die notwendige und sinnvolle Ernährungstherapie droht noch weiter vom unzureichenden Ist-Niveau abzufallen. Auf Initiative der Deutschen Gesellschaft für Ernährungsmedizin (DGEM) fand daher am 14. Juni 1999 in Bonn ein Expertengespräch zum Thema «Enterale Ernährungstherapie» statt. Ziel dieses Gespräches war es, den aktuellen Kenntnisstand bezüglich der enteralen Ernährungstherapie und ihrer Anwendung im medizinischen Alltag unter Berücksichtigung der Sichtweise der verschiedenen Disziplinen und Fachorganisationen einvernehmlich zu definieren. Auf dieser Grundlage kann die derzeitige Situation kritisch bewertet und entsprechende Forderungen für zukünftige Entwicklungen abgeleitet werden.

1 Teilnehmer: Dr. Michael Adelmann, leitender Oberarzt Klinikum Weilmünster; Dr. Jürgen Bausch, Vorsitzender der Kassenärztlichen Vereinigung Hessen, Frankfurt; Prof. Dr. Wolfgang F. Caspary, Johann Wolfgang Goethe-Universität Frankfurt, Direktor Medizinische Klinik II, Abteilung für Gastroenterologie; Dr. Wolfgang Ingenhag, Bundesverband der Betriebskrankenkassen, Abteilung Medizin, Essen; Prof. Dr. Karl-Walter Jauch, Präsident der Deutschen Gesellschaft für Ernährungsmedizin (DGEM), Regensburg; Siegrid Kalde, Deutscher Berufsverband der Diätassistentinnen, Ernährungsteam Universität Düsseldorf; Helmut Laschet, Stellvertretender Chefredakteur der Ärzte-Zeitung, Redaktionsleitung Gesundheitspolitik, Neu-Isenburg; Prof. Dr. Christian Löser, Universitätsklinikum Kiel, leitender Oberarzt Klinik für Allgemeine Innere Medizin; Prof. Dr. Manfred J. Müller, Christian-Albrechts-Universität zu Kiel, Direktor Institut für Humanernährung und Lebensmittelkunde; Prof. Dr. Peter Oberender, Universität Bayreuth, Lehrstuhl für Volkswirtschaftslehre, Forschungsstelle für Gesundheitsökonomie und Sozialrecht, Bayreuth; Prof. Dr. Peter Stehle; Rheinische Friedrich-Wilhelms-Universität Bonn, Direktor Institut für Ernährungswissenschaft; Prof. Dr. Elisabeth Steinhagen-Thiessen, Universitätsklinikum Charité, Lipidambulanz, Apherese, Diabetik und Ernährungsmedizin, Ärztliche Leiterin Evangelisches Geriatriezentrum, Berlin; Dr. Roman Stiller, Städtisches Krankenhaus München-Schwabing, Institut für Diabetologie; Dr. Klaus Wahle, Berufsverband der Allgemeinärzte Deutschlands - Hausärzteverband (BDA), Lehrbeauftragter an der Westfälischen Wilhelms-Universität, Münster; Dr. Hansjörg Werner, Chefarzt Klinik für Geriatrie, Evangelisches Krankenhaus Elisabethenstift, Darmstadt.

Berücksichtigt wurde die schriftlichen Stellungnahme von Dörte Friedrich, Deutscher Berufsverband für Pflegeberufe, Eschborn.
Das vorliegende Papier faßt die Ergebnisse des Expertengesprächs zusammen und berücksichtigt abschließend die schriftlichen und mündlichen Stellungnahmen aller Teilnehmer.

\section{Mangelernährung}

Die Malnutrition ist Ausdruck einer Unterversorgung an Energie, Protein, essentiellen Fettsäuren, Vitaminen, Mineralstoffen oder Spurenelementen. Studien zufolge sind zwischen 30 und $60 \%$ aller Patienten im Krankenhaus fehl- bzw. mangelernährt. Unter Berücksichtigung des altersspezifischen Normbereichs weisen sie eine unzureichende Energie-, Protein- (Serumkonzentration von Gesamtprotein, Albumin, Transferrin) und Vitaminzufuhr (Blutwerte von Vitamin A und C) auf. In Deutschland gibt es bislang keine wissenschaftlich begründeten Daten zum Energiebedarf. Die Bestimmung des individuellen Nährstoffumsatzes kann über eine indirekte Kalorimetrie erfolgen. Aufgrund des Geräteaufwands wird dies jedoch nur selten in Kliniken durchgeführt. Ein regelmäßiges Monitoring der Nährstoffaufnahme ist daher dringend erforderlich.

Ursachen für eine Mangelernährung sind:

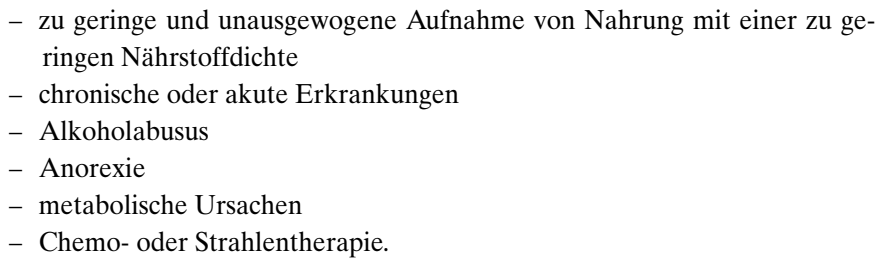

\section{Mangelernährung in der Geriatrie}

Der Anteil der mangelernährten geriatrischen und neurologischen Patienten im ambulanten Bereich beträgt 5-10\%. Hochbetagte geriatrische Patienten leiden häufiger unter einer Mangelernährung als gesunde jüngere Senioren und gesunde Gleichaltrige. Besonders häufig tritt die Malnutrition jedoch bei Patienten in geriatrischen Kliniken, in Einrichtungen der Altenpflege und bei Demenzkranken auf. 40\% der Demenzkranken erleiden einen Gewichtsverlust von $2-5 \%$ jährlich, der insbesondere bei einem niedrigen Ausgangsgewicht ernsthafte gesundheitliche Folgen nach sich ziehen kann.

Ursachen aus medizinischer Sicht sind neben den oben genannten:

- Erkrankungen der Zähne und des Mundes

- Auslassen von Mahlzeiten

- Polypharmakotherapie

- Schwierigkeiten bei der Nahrungsaufnahme (Schluckbeschwerden)

- mangelnder Appetit aufgrund schlechter Sehfähigkeit, mangelnder Geschmacks- und Geruchssinn

$\begin{array}{ll}\text { KARGER } & \text { @ 2000 S. Karger GmbH, Freiburg } \\ \begin{array}{l}\text { Fax }+497614520714 \\ \begin{array}{l}\text { E-mail Information@Karger.de } \\ \text { www.karger.com }\end{array}\end{array} & \text { Accessible online at: } \\ \text { www.karger.com/journals/cga }\end{array}$


- Mobilitätseinschränkungen aufgrund geringer körperlicher Fitneß und mangelnder Beweglichkeit.

\section{Folgen der Mangelernährung}

Die Nährstoffaufnahme dient nicht nur der Bedarfsdeckung für die Erhaltung der Organfunktionen, sondern zusätzlich dem Energiebedarf für körperliche Leistungen, Körperwachstum sowie Differenzierung der Gewebe und Organe. Der Einfluß der Ernährung auf Wohlbefinden, Gesundheit und Genesung ist daher bei geriatrischen Patienten, Kindern, Tumorpatienten, Intensivpatienten und Patienten mit chronischen Erkrankungen besonders groß. Folgen einer nicht adäquaten und bedarfsgerechten Ernährung sind:

- Beeinträchtigung des Stoffwechsels

- Störungen der Organaktivität, Organatrophie

- Akzeleration der Katabolie mit progredientem Gewichtsverlust bei schwerkranken Patienten

- Muskelschwäche, verminderte Belastbarkeit, Apathie und allgemeine Schwäche

- verringerte Mobilität mit erhöhter Sturzgefahr und Folgen wie Frakturen etc.

- Erhöhung von Mortalität und Morbidität

- vermehrte Krankenhausaufenthalte

- Beeinträchtigung der Immunabwehr und erhöhte Infektanfälligkeit

- Entstehung von Wunden und verzögerte Wundheilung, besonders bei bettlägerigen oder abgemagerten Patienten

- Erhöhung postoperativer Komplikationsraten

- erhöhter Verbrauch von Therapeutika/Arzneimitteln, z. B. Antibiotika

- verzögerte Rehabilitation nach akuten Erkrankungen

- Reduktion der Lebensqualität der Patienten

- Beeinträchtigung des Verlaufs chronischer Erkrankungen z. B. bei chronischer Lebererkrankung

- Reduktion der Therapietoleranz, z.B. bei Tumorpatienten mit Radio-, Chemo- oder operativer Therapie

- bei Kindern gestörte körperliche Entwicklung wie gestörtes Längenwachstum, verzögerte Pubertätsentwicklung, gestörte Differenzierung und Funktionen einzelner Organsysteme und des Nervensystems, gestörte geistige Entwicklung

- bei Kindern erhöhte Wahrscheinlichkeit für koronare Herzerkrankungen im Erwachsenenalter.

\section{Ernährungstherapie zur Behebung der Malnutrition}

Grundsätzlich sollten die Patienten nach Möglichkeit mit einer ausgewogenen Normal- oder Vollkost oral ernährt werden, solange diese den Energie- und Nährstoffbedarf des Patienten sicherstellt und vom Patienten in ausreichender Form aufgenommen und ihre Inhaltsstoffe resorbiert werden können. Hierbei bieten sich folgende Möglichkeiten zur Verbesserung der Ernährungssituation:

- konsequentes Erfassen des Ernährungsstatus

- Ausschalten von Risikofaktoren

- energiereiche Wunschkost

- spezielle Kost bei Kau- und Schluckstörungen

- häufigere kleinere Mahlzeiten

- Qualitätskontrolle der Nahrung

- Beobachtung der Eßgewohnheiten und des Eßverhaltens

- Dokumentation der Nahrungsaufnahme
- Hilfeleistung bei der Nahrungsaufnahme

- Einsatz von Hilfsmitteln

- regelmäßige Gewichtskontrolle

- Beachtung von Pflegemaßnahmen, die die Ernährung betreffen: ruhige Atmosphäre, aufrechte Sitzhaltung, Zuwendung

- sorgfältiges Erfassen von Störungen der Ernährung und ihrer Folgen.

Eine künstliche Ernährung ist dann indiziert, wenn der Patient nicht mehr ausreichend essen/schlucken kann, darf oder will oder die Nahrungsbestandteile nicht in genügendem Maße resorbiert werden können.

\section{Enterale Ernährung}

Die künstliche Ernährung umfaßt die parenterale und die enterale Ernährung. Ist die Funktion des Magen-Darm-Trakts stark eingeschränkt, kommt eine parenterale Ernährung zum Einsatz. Bei der enteralen Ernährung erfolgt entweder eine orale Ernährung über Trinknahrung oder die Ernährung über eine Sonde. Ißt der Patient bei normaler Funktion des Magen-Darm-Trakts nicht ausreichend und nimmt er auch trotz Verabreichung der Nahrung zu wenig, bedarf es einer Ergänzungsdiät in Form von Trinknahrung. Ist eine orale Aufnahme überhaupt nicht möglich, bedarf es einer Sondenernährung. Dabei wird die Nahrung in der Regel kontinuierlich mit einem Pumpensystem über einen Zeitraum von mehreren Stunden appliziert. Die Sondenführung erfolgt am häufigsten durch die Bauchdecke direkt in den Magen (perkutane endoskopische Gastrostomie, PEG), seltener nasogastral oder über Feinnadelkatheterjejunostomie (FKJ) direkt in den Dünndarm. Somit können auch Patienten mit starker Beeinträchtigung des Gastrointestinaltrakts über längere Zeiträume qualitativ ausreichend ernährt werden. Die orale Nahrungsaufnahme kann und soll auch bei festsitzender Sonde trainiert werden (Schlucktraining), sofern es die Grunderkrankung erlaubt.

Die enterale Ernährung ist die effektivste und risikoärmste Form der künstlichen Ernährung. Sie bietet gegenüber der parenteralen Ernährung viele medizinische Vorteile. Sie ist weiterhin physiologischer, weniger invasiv und birgt weniger Komplikationen. Ist eine enterale Ernährung aufgrund des Gesundheitszustands des Patienten möglich, ist sie heute die Methode der Wahl.

\section{Indikationen der enteralen Ernährungstherapie}

Durch die europäischen Richtlinien über diätetische Lebensmittel für besondere medizinische Zwecke ist der medizinische Charakter der bilanzierten Diäten definiert. Ihre Verordnung ist derzeit durch die Arzneimittelrichtlinie 17.1.i geregelt.

(Indikationen: Morbus Crohn, Kurzdarmsyndrom, stark Untergewichtige mit Mukoviszidose, Patienten mit chronisch terminaler Niereninsuffizienz unter eiweißarmer Ernährung, Patienten mit konsumierenden Erkrankungen, alte Menschen im Zustand der Unter-/Mangelernährung und medizinisch indizierte Sondennahrung.)

Den hohen Zuwachsraten der Verordnungen zufolge ist der Bedarf an enteraler Ernährungstherapie hoch. Sozialrechtlich ist die Sondenernährung für die Verordner nach $\S 31$ SGB V budgetrelevant. Der niedergelassene Arzt, insbesondere der Hausarzt, ist aufgrund 
dieses Budgetdrucks nicht an einer Mengenausweitung interessiert, sondern richtet sich am Bedarf der Patienten aus.

Ziel der enteralen Ernährung ist die Vermeidung des Hungerzustands mit den Folgen von Infektionen und Tod, die Behebung manifester und drohender Malnutrition (nutritive Effekte) sowie die Begünstigung des natürlichen Verlaufs oder der Therapie einer spezifischen Krankheit (therapeutische Effekte).

Eine enterale Ernährungstherapie wird momentan in den Fachdisziplinen Innere Medizin, Gastroenterologie, Geriatrie, Diabetologie, Chirurgie, Onkologie, Pädiatrie, HNO, ZMK, Pulmonologie, Neurologie und Immunologie eingesetzt bei:

- manifester Fehlernährung (Malnutrition); BMI $\leq 18,5 \mathrm{~kg} / \mathrm{m}^{2}$

- neurologischen Erkrankungen wie z. B. Schlaganfall und neurologisch bedingten Schluckstörungen

- chirurgischen Patienten nach Operation (gut ernährte Patienten, die mehr als 10-14 Tage nicht essen können, unterernährte Patienten, die sich einer elektiven Operation unterziehen)

- Patienten mit Trauma (Unfall, Kopfverletzung, Verbrennungen)

- kritisch kranken Patienten auf Intensivstationen

- Patienten mit Mund-, Rachen-, Kehlkopf-, Speiseröhren-, Magen- oder Dünndarm-Tumoren

- Patienten mit schweren Erkrankungen des Verdauungstrakts wie Kurzdarm-Syndrom, entzündlichen Magen-Darm-Erkrankungen, chronisch intestinaler Pseudoobstruktion oder Malassimilationssyndrom

- Patienten mit Knochenmarkstransplantation und intensiver Chemotherapie

- Patienten der Strahlentherapie

- AIDS mit Wasting-Syndrom

- Stoffwechselerkrankungen

- Infektionserkrankungen

- pulmonalen Erkrankungen bei Kindern, Jugendlichen und Erwachsenen

- Alten und Verwirrten mit psychoorganischen Veränderungen.

Die momentane Anwendung der enteralen Ernährungstherapie (siehe oben) zeigt, daß die Arzneimittelrichtlinien (17.1.i) der Problematik im ambulanten Bereich, insbesondere was den tatsächlichen Bedarf anbetrifft, nicht gerecht werden. Ihre Überarbeitung ist im Hinblick auf den hohen Anteil geriatrischer Patienten dringend erforderlich.

\section{Nahrungen}

Bei der enteralen Ernährung können selbsthergestellte oder industriell gefertigte Trink- und Sondennahrungen zum Einsatz kommen. Die selbsthergestellte Küchenkost (Küchensonde) wird jedoch den spezifischen Bedürfnissen des Patienten nicht gerecht, da die Inhaltsstoffe nicht dem geänderten Bedarf angepaßt (bilanziert) sind. Aufgrund weiterer Nachteile wie beispielsweise die Kontaminationsgefahr und der Risiken einer fehlerhaften Therapiesteuerung ist die Küchensonde daher medizinisch und ökonomisch indiskutabel und kann bei definierter medizinischer Indikation nicht verantwortet werden.

Bilanzierte Diäten sind industriell hergestellte Produkte mit einer standardisierten, definierten Zusammensetzung in bezug auf Nährstoffe (Fette, Proteine, Kohlenhydrate), Mineralstoffe, Spurenelemente und Vitamine. Sie richtet sich verbindlich nach den Definitionen und Standards der Diätverordnung (§14). Die bilanzierten
Diäten enthalten alle essentiellen und nichtessentiellen Nähr- und Wirkstoffe, die ein Patient zu einer optimalen Versorgung benötigt. $\mathrm{Zu}$ den bedarfsdeckenden bilanzierten Diäten gehören die hochmolekularen, nährstoffdefinierten Diäten (NDD) und die niedermolekularen, chemisch definierten Diäten.

Weitere Vorteile der bilanzierten Diäten:

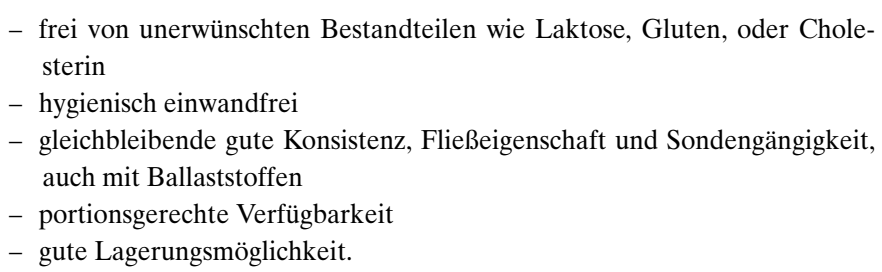

Die vielfältigen Darreichungsformen und Applikationsmöglichkeiten der bilanzierten Diäten ermöglichen eine individuelle adäquate Ernährungsstrategie und erleichtern die Umsetzung im ambulanten und stationären Alltag.

\section{Nutritive Effekte der Ernährungstherapie}

Wissenschaftliche Studien haben gezeigt, daß eine frühzeitig angewandte Ernährungstherapie die Nährstoffzufuhr steigert und positive Effekte auf den Ernährungszustand sowie den Therapie- und Heilungsverlauf hat. Die enterale Ernährung per se schützt den Körper z. B. vor dem Eindringen von Erregern über den Darm und vermindert so postoperative Infektionen. Nach neuen Erkenntnissen ist eine nährstoffreiche Nahrungsergänzung in flüssiger Form (Trinknahrung) derzeit die effektivste Art, bei Patienten die Nährstoffaufnahme zu steigern und den Ernährungszustand zu verbessern. Durch die tägliche Zufuhr energiereicher Trinknahrung werden beispielsweise die mittlere Überlebensrate bei Mukoviszidosepatienten, die Muskelkraft bei kurativ rezesierten Magenkarzinompatienten sowie die Gehfähigkeit bei Patienten mit Schenkelhalsfraktur positiv beeinflußt. Eine adaptierte Trink- oder Sondennahrung kann daher in der Geriatrie in bestimmten Fällen ein wichtiger Bestandteil kurz-, aber auch langfristiger therapeutischer Interventionen bei manifester und drohender Malnutrition sein.

\section{Therapeutische Effekte}

Zahlreiche experimentelle und klinische Studien haben den Nachweis erbracht, daß bestimmte Nährstoffe mehr als nur nutritive Wirkungen haben. Diese additiven Effekte beinhalten die Modulation des Immunsystems und neurologischer Funktionen sowie die Unterstützung der Funktion geschädigter Organe und Gewebe. Ein quantitativ und qualitativ kontrollierter Einsatz dieser Substrate im Rahmen einer maßgeschneiderten enteralen Ernährung für spezielle Indikationen wie Stoffwechselerkrankungen, metabolischen Streß, kardiopulmonare Dysfunktion, Lebererkrankungen und Niereninsuffizienz kann nachweislich den Heilungsverlauf günstig beeinflussen. Darüber hinaus ist beispielsweise eine eiweißreiche Ernährung für die Prävention und Therapie von Dekubiteralulzera unverzichtbar. Bilanzierte Diäten werden daher mit diesen «nutraceutics», das 
sind Nährstoffe mit pharmakologischen Eigenschaften wie z. B. Arginin, Glutamin, Omega-3-Fettsäuren, Nukleotiden und kurzkettigen Fettsäuren bzw. Ballaststoffen und Vitaminen, angereichert. Durch eine spezielle diabetesadaptierte bilanzierte Trink- und Sondendiät werden postprandial deutlich geringere Blutglukose und Seruminsulinspiegel erzielt. Dies führt zu einer rascheren Rekonvaleszenz und einer deutlich verbesserten Stoffwechselführung, bei der auf keine zusätzliche Medikation zurückgegriffen werden muß. Die enterale Ernährung ist so auch Therapie der Grunderkrankung. Sie ist im Kontext der Gesamttherapie ein fester und unverzichtbarer Bestandteil der Behandlung Schwerkranker.

\section{Ernährungstherapie als (haus)ärztliche Aufgabe}

Die Ernährungsberatung und die Therapie der verschiedenen Formen der Unter- und Mangelernährung und ihrer Folgen nehmen einen breiten Raum der ärztlichen Tätigkeit ein. Diese reichen von der Diagnose, der Identifikation von Ursachen und Risikofaktoren für die Ernährungsprobleme bis hin zur Anordnung und Durchführung von Therapiemaßnahmen. Die enterale Ernährungstherapie wird häufig in der Klinik verordnet und eingeleitet. Durch den hohen Anteil an Karzinom- und geriatrischen Patienten findet sie vermehrt in der hausärztlichen Praxis Anwendung.

Die ambulante Medizin ist auf die enterale Ernährungstherapie angewiesen. Die demographischen und sozialen Entwicklungen und auch die politisch gewollte Verlagerung von der stationären hin zur ambulanten Versorgung verstärken diesen Trend. Eine Mengenausweitung der enteralen Ernährungstherapie ist somit unter der momentanen Sozialgesetzgebung unvermeidbar. In der Praxis steht jedoch die Verordnung der enteralen Ernährungstherapie als integraler Bestandteil eines Behandlungskonzepts auch bei medizinisch festgestellter Indikation in Diskussion. Im ambulanten bzw. Pflegebereich hat sich aufgrund des immer größer werdenden Anteils alter Menschen in der Bevölkerung auch der Anteil der Patienten mit Sondenernährung und daher der Markt für die Sondenernährung kontinuierlich vergrößert. Aus diesem Grund sind Leitlinien zur Verordnung der enteralen Ernährungstherapie dringend erforderlich. Die Formulierung sollte den medizinischen Fachgesellschaften vorbehalten sein. Danach ist das Zertifizierungsverfahren der Ärztlichen Zentralstelle für die Qualitätssicherung (ÄZO) bei der Kassenärztlichen Bundesvereinigung und der Bundesärztekammer in die Wege zu leiten. Diese Leitlinien könnten die Grundlage für Maßnahmen zur Budgetsteuerung bilden. Dennoch sollte dem Arzt in jedem Fall weiterhin die Indikationsstellung und Verordnungshoheit der Ernährungstherapie obliegen.

\section{Verzahnung der ambulanten und stationären enteralen Ernährungstherapie}

Die enterale Ernährungstherapie ist wenig belastend für den $\mathrm{Pa}$ tienten und aufgrund der weiterentwickelten Technik und konfektionierten Darreichungsformen einfach zu handhaben. Sie kann nach Anleitung vom Pflegepersonal, dem Patienten selbst oder seinen Angehörigen auch zu Hause durchgeführt werden. Gesundheitliche Risiken können nahezu vernachlässigt werden. Die heimenterale Ernährungstherapie fördert die Lebensqualität und somit auch die Genesung des Patienten, da er sich im vertrauten Umfeld befindet und je nach gesundheitlicher Beeinträchtigung am normalen Alltag teilnehmen kann. Daher sollte ihr Einsatz zusammen mit dem Erkrankten frühzeitig erwogen werden. Eine Entscheidung für eine bestimmte Form der Ernährungstherapie muß in jedem Fall umfeldbezogen sein. Für eine konsequente Weiterführung der Ernährungstherapie im ambulanten Bereich ist die Information des Hausarztes über Ernährungsprobleme, Therapie usw. die Grundvoraussetzung.

Der niedergelassene Arzt verordnet und koordiniert die ambulante Ernährungstherapie. Je nach Indikation und Therapieform wird er durch ein multiprofessionelles Team von Fachärzten, Pflegefachkräften, Ernährungsberatern, Ernährungswissenschaftlern und Diätassistenten unterstützt. Die Fachpflegekräfte pflegen den Patienten, unterstützen ihn im Umgang mit der Therapie und stehen ihm bei Problemen im häuslichen Milieu zur Verfügung. Auch professionelle Anbieter erleichtern durch ihre Serviceleistungen in bezug auf Pflege, Lieferservice für Nahrungen, technischen Service usw. die Durchführung der enteralen Ernährungstherapie zu Hause. Durch regelmäßige gemeinsame Absprachen zwischen allen Beteiligten werden Defizite frühzeitig aufgedeckt; Korrekturen können rechtzeitig vorgenommen werden. Im Sinne einer optimalen und langfristigen Betreuung des Ernährungspatienten über die Zeit der Klinik hinaus ist eine engere Verzahnung zwischen stationärer und ambulanter Versorgung bei der heimenteralen LangzeitTherapie wünschenswert.

\section{Qualitätskontrolle der enteralen Ernährungstherapie}

Die enterale Ernährung ist eine uneingeschränkt anerkannte Therapieform und wird weltweit in der klinischen Praxis eingesetzt. Dennoch gibt es bislang weder eine Leitlinie für die Indikationsstellung und Qualitätssicherung der Versorgung noch eine einheitliche Aus- und Fortbildung der Ärzte in der Ernährungsmedizin. Die meisten Ärzte kennen nicht einmal die Instrumente zur Diagnose der Mangelernährung. Die Qualitätssicherung bleibt dem persönlichen Engagement des Arztes überlassen. Derzeit wird die Ernährungstherapie lediglich in der Ausbildung der Ökotrophologen und Ernährungswissenschaftler berücksichtigt. Das Curriculum Ernährungsmedizin der Bundesärztekammer, dessen Umsetzung den Landesärztekammern obliegt, die Fachkunde Ernährungsmedizin der Ärztekammern Schleswig Holstein und Niedersachsen sowie der Titel eines Ernährungsbeauftragten Arztes der Deutschen Akademie für Ernährungsmedizin sind erste Maßnahmen zur Qualitätssicherung in der Ernährungstherapie.

Zur Qualitätsverbesserung sollte das vorhandene Wissen zu den Grundlagen der Ernährung und der Ernährungstherapie bei Ernährungswissenschaftlern und Diätassistenten eingeholt und in die 
Therapiekontrolle integriert werden. Langfristig sollten ernährungsmedizinische Kurse im Lehrplan der Universitäten eingerichtet und die Approbationsordnung für Ärzte novelliert werden. Eine bundesweite Vereinheitlichung ist notwendig.

Der Verband der Diätassistenten - Deutscher Bundesverband führt seit 1993 in Zusammenarbeit mit der Deutschen Akademie für Ernährungsmedizin und der Deutschen Gesellschaft für Ernährung für Diätassistenten eine zertifizierte Weiterbildung zur «sondenbeauftragten Diätassistentin» durch. Diese sollten im Sinne einer Qualitätssicherung verstärkt genutzt werden.

Das Pflegepersonal trägt in besonderem Maße Verantwortung für die Ernährung des Patienten. Die Information des Pflegepersonals über die häusliche Ernährungssituation sind für den behandelnden Arzt ein ebenso wichtiges Indiz als Grundlage für die Verordnung einer enteralen Ernährung wie seine eigenen diagnostischen und therapeutischen Daten. Intensive und regelmäßig wiederholte Schulungen und Fortbildungen aller beteiligten Berufsgruppen sind daher dringend erforderlich. Es ist sinnvoll, sowohl im stationären wie auch im ambulanten Bereich speziell ernährungsmedizinisch geschulte Schwestern und ernährungsbeauftragte Ärzte zu bestellen, wie dies modellhaft an wenigen Kliniken praktiziert wird.

\section{Leistungsvergütung bei medizinischem und pflegerischem Personal}

Im ambulanten Bereich gibt es bislang für Ärzte keine Vergütungsziffer für die Ernährungstherapie. Eine ausreichende und adäquate Honorierung ist jedoch eine wesentliche Grundlage für eine gesicherte Qualität. Ein Arzt, der sich nach den in «Abschnitt Qualitätskontrolle in der enteralen Ernährungstherapie» geforderten Aus- und Weiterbildungen qualifiziert hat und die Diagnose und Therapie der Mangelernährung beherrscht, sollte daher für die mit der enteralen Ernährungstherapie verbundene Verantwortung mit einer entsprechenden Leistungsvergütung entlohnt werden. Diese Forderung gilt auch für alle anderen die enterale Ernährungstherapie betreuenden Berufsgruppen.

\section{Erstattungsfähigkeit der enteralen Ernährungstherapie}

Die ambulante Ernährungstherapie als häusliche Krankenpflege hat ihre gesetzliche Grundlage in $\$ 37$ SGB V. Im Einzelfall kann in der Praxis die Abgrenzung zu den Leistungen der Pflegeversicherung zu Schwierigkeiten führen. Die Verabreichung von Sondennahrung fällt unter die Grundpflege im Rahmen des $\S 37$ SGB V bzw. dann gegebenenfalls unter die Pflegeversicherung (SGB XI), wenn der Pflegefall bereits eingetreten ist. Sonden und weitere notwendige Materialien zur Durchführung der enteralen Ernährung sind Hilfsmittel (Medizinprodukte) und werden von den Kostenträgern übernommen. Der Anspruch der Versicherten auf Erstattung der medizinisch indizierten bilanzierten Diäten er- gibt sich aus $\S 27$ und $\S 31$ SGB V in Verbindung mit der gültigen Fassung Nummer 17.1.i der Arzneimittelrichtlinien. Bis auf die entsprechende Zuzahlung sind diese Produkte für den Versicherten kostenfrei. In der Praxis wird die Erstattung hin und wieder von den Kostenträgern abgelehnt in der Annahme, es handle sich um Nahrungsmittel. Die daraufhin notwendigen Rücksprachen zwischen Kostenträgern, Hausärzten, Patienten und Pflegediensten bis zur endgültigen Klärung bzw. Kostenübernahme erfordern viel Zeit und Geduld und können den Patienten in finanzieller und gesundheitlicher Hinsicht beeinträchtigen.

Momentan wird nur ein Sondenpatient als Ernährungspatient gesehen. Auch nach Meinung der Kassenärztlichen Vereinigung besteht ein Defizit zwischen Rechtslage und medizinischer Erkenntnis. Sie empfiehlt den Ärzten, die supplementierende Trinknahrung bei intakter Schluckfähigkeit nicht zu verordnen. In Anbetracht dieser Situation sowie der geringen Leistungsvergütung und der Budgetierung der Verordnungen geraten daher Ärzte in Versuchung, die Patienten nur aufgrund der Ernährungstherapie in die Klinik zurückzuschicken. Da ein solches Verhalten absolut ungerechtfertigt ist, muß eine medizinisch notwendige enterale Ernährungstherapie (Trink- und Sondennahrungen) weiterhin zu Lasten der Leistungsträger verordnet werden können. Die Kostenerstattung darf nicht von der Applikationsform abhängig sein. Die supplementierende Trinknahrung muß als Therapieform anerkannt werden. Es müssen Wege gefunden werden, die schwierige Abgrenzung der konfektionierten Trinknahrung als Ernährungsbestandteil von der sinnvollen therapeutischen Anwendung im Krankheitsfall zu vereinfachen. Insgesamt bedarf es einer größeren Transparenz bezüglich der Kostenübernahme und einer Präzisierung der teilweise unzureichend definierten medizinischen Indikationen.

\section{Ökonomische Betrachtung}

Mangelernährung führt zu einer Kostensteigerung im Gesundheitswesen. Angesichts der zunehmenden Diskrepanz zwischen medizinisch sinnvoll Möglichem und Finanzierbarem muß die Voraussetzung für eine effektive und effiziente Therapie der Mangelernährung geschaffen werden. Bei der Entscheidung des Arztes für ein Ernährungsregime müssen neben medizinischen auch soziale, ethische und vor allem ökonomische Dimensionen beachtet werden; neben den direkten Kosten (Personal-, Sach- sowie Behandlungskosten), den indirekten Kosten (Verdienstausfall, Arbeitszeitverlust, Produktionsausfall, zukünftige Gesundheitsausgaben - z. B. durch eine höhere Lebenserwartung) sind auch die intangiblen Kosten und Nutzen (beispielsweise Glück, Freude, Angst, Trauer, Leid und vor allem Lebensqualität) zu berücksichtigen.

Eine adäquate, medizinisch indizierte enterale Ernährungstherapie gewinnt als Kosten- und Erfolgsfaktor hierbei eine immer größere Bedeutung. Ihr frühzeitiger Einsatz senkt die Zahl von Komplikationen, verkürzt die Anzahl und Dauer des stationären Aufenthalts, senkt die Kosten für Medikamente und Pflege, steigert die 
Therapietoleranz und verbessert die Lebensqualität und Prognose des Patienten. Die zunächst teuer erscheinende enterale Ernährungstherapie stellt sich so insgesamt als «kosteneffektiver» dar und ist damit wirtschaftlich.

Gegenüber der parenteralen Ernährungstherapie ergeben sich für die Applikation eines enteralen Regimes deutlich geringere Gesamtkosten. Die Kostendifferenz beruht vor allem auf den Unterschieden hinsichtlich der Laborkosten, Kosten für Nährlösungen, Installationen und Verbrauchsmaterialien sowie der Kosten für Pflegepersonal und Ärzte. Weitere Einsparungen ergeben sich durch die Möglichkeit, die Krankenhausaufenthalte durch eine ambulante enterale Ernährung zu verkürzen. Gegenwärtig wird jedoch das vorhandene Rationalisierungspotential der enteralen Ernährungstherapie in der Prävention und der kurativen Versorgung noch nicht genutzt. Um den gesamten Kosten-Nutzen-Effekt einer adäquaten Ernährung zu erfassen, sind ökonomische Evaluationsstudien notwendig.

\section{Zukünftige Strategien}

Die enterale Ernährungstherapie ist eine weltweit anerkannte Form zur Therapie von marginaler und manifester Malnutrition. Im medizinischen Alltag in Deutschland hat sie jedoch nicht den Stellenwert, der ihr auf der Basis des heutigen Wissens zusteht. Zur Verbesserung der momentanen Situation wurden unter den Gesichtspunkten der medizinischen Notwendigkeit, der Praktika- bilität und Finanzierbarkeit folgende Anforderungen an die zukünftige Strategie der enteralen Ernährungstherapie gestellt:

- standardisierte Erhebung des Ernährungszustands bei allen Patienten als obligatorischer Bestandteil der ärztlichen Untersuchung

- Erstellung eines definierten und verbindlichen Indikationskatalogs

- einheitliche Richtlinien für die Qualitätssicherung

- einheitliche Fort- und Weiterbildung als integraler Bestandteil des Medizinstudiums (Aufnahme der Ernährungsmedizin in den Lehrplan, Novellierung der Approbationsordnung)

- optimierte stationäre und ambulante Versorgung von Ernährungspatienten

- ernährungsbeauftragte Ärzte stationär/ambulant

- ernährungsbeauftragte Schwestern bzw. Anwendungsberater

- sondenbeauftragte Diätassistenten

- Einrichtung von Ernährungsteams an Schwerpunktkrankenhäusern

- leistungsgerechte Vergütung von Diagnose, Behandlung, Schulung und Betreuung im ambulanten und stationären Bereich über Komplettgebühren bzw. über die Entgelte

- kontrollierte Studien über den Effekt von Ernährungsmaßnahmen

- ökonomische Evaluationsstudien

- Maßnahmen zur Budgetsteuerung wie Analysen des Verordnungsverhaltens und der Marktbewegungen sowie Informationsveranstaltungen zu Budgetfragen bei Ärzten und Pharmaunternehmen

- Mitgliedschaft der DGEM als Sachverständiger der Kommission für Ernährungstherapie des Instituts für Arzneimittelverordnung (Positivliste)

- Anhörung der DGEM und Einfließen ihrer Position in die Entscheidungen des Bundesausschusses der Ärzte und Krankenkassen

- Ernährungsmaßnahmen als fester Bestandteil eines multidimensionalen Therapieprogramms bei geriatrischen und Tumorpatienten (mit Entscheidungsbaum).

\section{Weiterführende Literatur}

1 Anderson JW, Akanji AO: Dietary fiber - an overview. Diabetes Care 1991;14:1126-1131.

2 Haslbeck M, Stiller R, Niederreiter B: Günstige metabolische Wirkungen einer kohlenhydratmodifizierten, bilanzierten Diät bei Typ-II-Diabetes. Aktuel Ernährungsmed 1995;20:215-220.

3 Letson AP, Price LS: Health, aging and nutrition: An overview. Clin Geriatr Med 1987;3:253.

4 Huth K: Parenterale Ernährung und Sondenkost; in Huth K, Kluthe R (Hrsg): Lehrbuch der Ernährungstherapie. Stuttgart, Thieme, 1986 , pp 228-256.

5 Otto C, et al: Influence of fiber, xylitol and fructose in enteral formulas on glucose and lipid metabolism in normal subjects. Clin Invest 1993; 71:290-293.
6 Puchstein C: Bilanzierte Ernährung; in Lawin P (Hrsg): Praxis der Intensivbehandlung. Stuttgart, Thieme, 1994, pp 493-507.

7 Rupp P, Haslbeck M: Die Bedeutung der Ballaststoffe in der enteralen, künstlichen Ernährung. Krankenpflege J 1986;24:50-52.

8 Schlierf G, et al: Fehlernährung bei geriatrischen Patienten: Die Bethanien-Ernährungsstudie. Z Gerontol 1998;22:2-5.

9 Seiler WO: Hohes Vorkommen von Malnutrition bei kranken Betagten. Ernährungsumschau 1999;46:168.

10 Stürmer W, et al: Favourable glycaemic effects of a new balanced liquid diet for enteral nutrition results of a short-term study in 30 type II diabetic patients. Clin Nutr 1994;13:221-227.
11 Tkatch L, et al: Benefits of oral protein supplementation in elderly patients with fracture of the proximal femur. J Am Coll Nutr 1992;11:519.

12 Tucker HN, Miguel SG: Cost containment through nutrition intervention. Nutr News 1996; 54:111-121.

13 Vellas B, Riveniere SS, Fi J: Weight Loss and Eating Behaviour in Alzheimer Patients. Paris, Serdi, 1998. 